\title{
Frequency Dependence of Penetration through Gaps into Buildings at mmWave
}

\author{
Tim W. C. Brown ${ }^{1}$, Ruhan Xie ${ }^{1}$, Pat Chambers ${ }^{2}$ \\ ${ }^{1}$ Institute for Communication Systems, University of Surrey, Guildford, Surrey, UK, tim@brown76.name \\ ${ }^{2}$ Institute of Sensors, Signals \& Systems, Heriot Watt University, Edinburgh, EH14 4AS, UK
}

\begin{abstract}
Measurements in the range of $10-20 \mathrm{GHz}, 22$ $30 \mathrm{GHz}$ and $50-67 \mathrm{GHz}$ are presented in this paper that show the benefit of using gaps in building infrastructure to substantially improve the penetration loss in the order of $10 \mathrm{~dB}$ or more. Increasing the frequency substantially improves the opportunity to penetrate through the same size gap as it becomes electrically larger. The measurement setup used in this work involves the use of a ground floor infrared reflector glass door whereby the effect of the gaps can be compared both by closing the door and sealing the gaps with a conductor in order to identify the difference in penetration. Simulations were also carried out to verify the waveguiding and standing wave effects in the gaps.
\end{abstract}

Index Terms-antenna, propagation, measurement.

\section{INTRODUCTION}

The required data demands of $1 \mathrm{Gbits} / \mathrm{s}$ are exceeding the capacity available from existing spectrum below $6 \mathrm{GHz}$, which is drawing attention to mmWave bands above this frequency up to $100 \mathrm{GHz}$. Though these bands have been of interest for over two decades, they have mainly focused on two areas, namely backhaul links for packet microwave and or for device to device applications indoors, which can tolerate the excessively high path loss at such frequencies [1]. Only recently has interest drawn to using these bands of spectrum for mobile devices, more specifically for small cell access. It is also desirable that access points are placed outdoors such that they can penetrate into buildings to enable simpler implementation compared to indoor installations [2]. A significant concern, however, is the excessive penetration losses at such frequencies that will require substantial power transmissions so it is important to understand such characteristics.

Whether the scenario is penetrating in a line of sight (LOS) or non line of sight (NLOS), the penetration loss is particularly severe where infrared reflector (IRR) glass is used with several layers of glazing metal oxide coatings to reflect infrared rays away from a building [3]. High levels of attenuation are therefore expected since mmWave frequencies are close to the infrared range of the electromagnetic spectrum. Measured penetration losses at mmWave in the literature [4][5] agree with this showing over $30 \mathrm{~dB}$ at $28 \mathrm{GHz}$ for IRR glass even in LOS, while standard glass was reported at less than $4 \mathrm{~dB}$.

Penetration loss through IRR glass is already substantial in a LOS case but in a realistic NLOS case it will also be necessary to factor in further losses from an outdoor base station to an indoor mobile in the general case. There are two possible propagation solutions illustrated in Fig. 1. The antenna beams of the base station and mobile could be aligned as shown in the green case to rely on the diffraction around the near edge of the window, while the alternative is that the beams align to the case shown in blue whereby they rely on reflection from the far edge of the window.

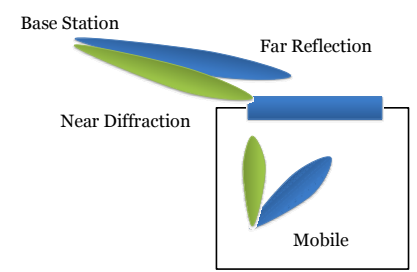

Fig. 1 - Diffraction effect around a window edge and reflection off a far edge at mmWave

This paper investigates through measurement, ranging from $10 \mathrm{GHz}$ through to $67 \mathrm{GHz}$, the difference in penetration loss when comparing diffraction loss off a near edge and reflection off a far edge of a window. Additionally, the measurement setup allows the opportunity to show how gaps in infrastructure are proven to make a substantial difference to the penetration loss where it can improve in the order of $10 \mathrm{~dB}$.

\section{MEASUREMENT SETUP}

The chosen measurement location was a ground floor room opening out to a courtyard area with a large IRR glass door based on a ground floor room illustrated in Fig. 2 (a). This enabled measurements to be carried out in a practical way and enable ease of calibration. Such measurements are less practical with many existing windows that only open partially and are positioned in awkward locations. Hence the door used in this case is suitably representative of a window using the same nature of glass.

The measurement setup using horn antennas, cables and a network analyser is shown by a plan view illustrated in Fig. 2 (b). Two cases are defined as the "Near" case, relying on diffraction around the near edge, and the "Far" case, relying on reflection off the far edge of the door frame. As shown in Fig. 2 (b), the propagation paths begin at a transmitter horn antenna outside of the building and arrive at a receive horn antenna inside. The antennas are orientated to the Far and 
Near cases by appropriately pointing their beams towards the respective ends of the door frame. Additionally a power amplifier is used at the transmit end to enable sufficient dynamic range for the substantial penetration losses to be measured.

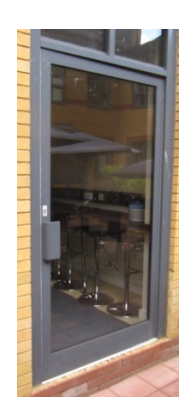

(a)

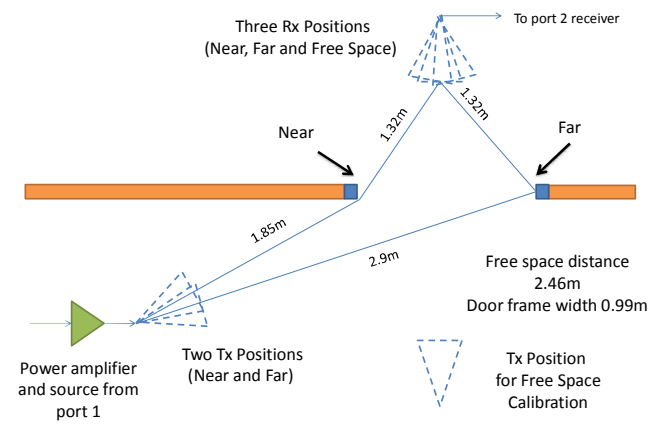

(b)
Fig. 2 Diagram of the measurement setup to measure the near and far edges

As shown in Fig. 2 (b), the horn antennas were connected to a vector network analyser in order to measure the penetration losses. Three sets of frequency range were used: $10-20 \mathrm{GHz}, 22-30 \mathrm{GHz}$ and $50-67 \mathrm{GHz}$. Different horn antennas were used in each frequency band whereby they had a gain of at least $18 \mathrm{dBi}$ and a beamwidth ranging from $15^{\circ}$ to $23^{\circ}$ depending on the frequency. In all cases the horns were orientated to have vertical polarisation.

To achieve maximum dynamic range in the measurements, the power level at the amplifier output was set to $10 \mathrm{dBm}$ in the $10-20 \mathrm{GHz}$ case, $20 \mathrm{dBm}$ in the $22-$ $30 \mathrm{GHz}$ case and $15 \mathrm{dBm}$ in the $50-67 \mathrm{GHz}$ case. However, given the excessive cable losses in the latter case, an additional $50-67 \mathrm{GHz}$ amplifier was used whereby one was directly feeding the transmitter horn and an additional one was placed at the start of the feeder cable to maximize the power transmitted into the antenna. Using the amplifier at the transmitter not only helped to compensate losses due to the free space separation and cable loss to improve dynamic range but no further additive noise was created in the system that would otherwise occur if an amplifier was placed at the receiver. A resolution bandwidth of $10 \mathrm{~Hz}$ was set on the network analyser to minimise the received noise floor. This ensured the capability to measure the deep frequency selective fades through the gaps across the band. Furthermore 3000 samples were set on the network analyser in order that there was sufficient sampling in the frequency domain every $2.67-5.67 \mathrm{MHz}$ resulting in a maximum measurable delay of $176-375 \mathrm{~ns}$, which is well beyond the maximum excess delay found to be below 100ns in the penetration region, while such delay taps would be captured within the system dynamic range.

It can be further observed in Fig. 3 (a), where the door is opened slightly ajar, that small gap of $10 \mathrm{~mm}$ is present complete with textiles to cushion impact when the door closes. On the other side of the door where it is hinged, another gap of $8 \mathrm{~mm}$ was present constructed similarly with a cushion effect and a ridge outer frame shown in Fig. 3 (b). This effectively created the gaps in building infrastructure as a test case for this measurement.

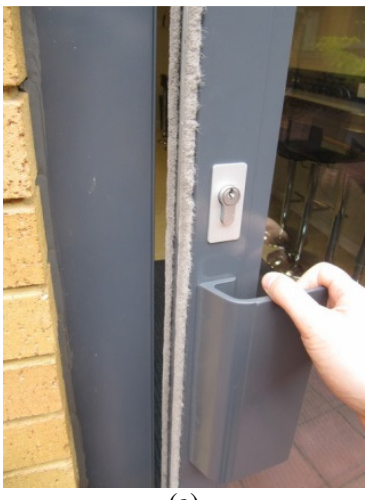

(a)

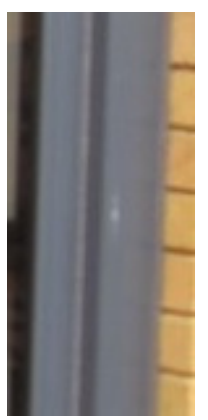

(b)
Fig. 3 - Photograph of the (a) opening gap at the near end and (c) hinge gap in the far end with ridge frame

It can be further observed in Fig. 2 (b) that the transmit antenna horn was also placed to directly face the receiver horn at the same height for purposes of carrying out a free space calibration measurement. The receiver antenna was fixed $1.27 \mathrm{~m}$ from the door and the transmit antenna was placed directly opposite a further $1.19 \mathrm{~m}$ away hence the total distance was $2.46 \mathrm{~m}$. This enabled a free space through calibration of scattering parameter $s_{21}$ to be taken, in order to calibrate out the cable losses and group delay of the amplifier as well as free space loss. This free space measurement could then be used as a reference point from which any penetration measurements could be calibrated. It should be noted that the transmit and receive antennas in this position are placed more than $1 \mathrm{~m}$ away from the glass door, which at such frequencies ensures that the free space was calibrated in the far field of both antennas above the Rayleigh distance [6] for all frequencies measured. It is also necessary to be as close as possible in order to minimise the magnitude of any scattered paths on the edge of the window pane. At these distances, the maximum antenna beamwidth of $23^{\circ}$, with a corresponding $10 \mathrm{~dB}$ beamwidth of $40^{\circ}$, means that any rays reflecting off the side of the door will be more than $20 \mathrm{~dB}$ less than the direct path, which will have negligible effect on the free space calibration. Once a calibration measurement had been undertaken in free space, the antennas were re-positioned to undertake Near and Far Measurements. By calculating the extra distances moved from the free space calibration to the measurement positions (as noted in Fig. 2 (b)), the corresponding change in free space path loss, can be calibrated out of the extra loss due to both change in distance and additional penetration loss measured with the network analyser. Therefore, after calibration, only the actual measurement of the penetration loss in the Far and Near case remained.

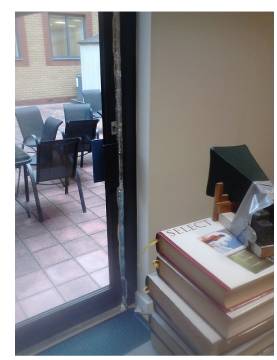

Fig. 4 Photograph of measurements conducted through the door from inside with a seal applied

As noted in Fig. 3, both the Far and Near scenarios had a physical gap in the door frame and in order to evaluate the 
penetration loss with and without gaps for comparison, aluminium tape was used to block the gap and form the case without gaps, which was possible because the thickness exceeded the skin depth at the frequencies used. An example case with the aluminium tape seal is shown in Fig. 4 where the Near case is seen from inside.

\section{MEASUREMENT RESUlTS}

The calibrated measurement results of penetration loss for the Near and Far scenarios, both when the door is closed, then sealed are compared in Fig. 4 in the frequency domain for the $10 \mathrm{GHz}$ to $20 \mathrm{GHz}$ range. It should first be noted that in the near case, though not shown in this paper it was found that by analysing the power delay profile from taking a Fourier transform of measured data in the frequency domain, there was initially a diffraction loss of approximately $27 \mathrm{~dB}$ on the direct path when the door was open. This is consistent with what would be expected from the well known knife edge diffraction model at the centre frequency [6]. When the door was closed, an additional loss of $6 \mathrm{~dB}$ resulted, but notably when the door was sealed, a further $12 \mathrm{~dB}$ increase in loss was reached. Therefore the total attenuation with the seal was $45 \mathrm{~dB}$ or only $33 \mathrm{~dB}$ without the seal. Clearly the gap in the door makes a significant improvement to the propagation when the beams are aligned accordingly and is highly desirable for such excessive loss. Given the substantially high penetration loss of the IRR glass, these small air gaps are beneficial to exploit.

By analysing the frequency domain data for both Near and Far cases in Fig. 4, it can first be observed that at higher frequencies above $14 \mathrm{GHz}$, there is a consistent difference of around 20dB observed between the sealed and non sealed (or closed) measurements. This is true in both the Near and Far cases. However, below $14 \mathrm{GHz}$ going down towards $10 \mathrm{GHz}$, in both Near and Far cases, the non sealed and sealed measurements converge such that the seal has no material effect below $11 \mathrm{GHz}$. This can be explained in part by a waveguiding effect in the door gap similar to that of a transverse electric $\left(\mathrm{TE}_{10}\right)$ mode waveguide cut off frequency [7] forming a frequency selective surface such that any frequency below the cut off will not propagate through the gap. The gap in the Near case has a cut off frequency of $15 \mathrm{GHz}$, hence the results are consistent with the theoretical expectation that propagation through the gap would be weak below this frequency and hence adding a seal makes little or no difference.

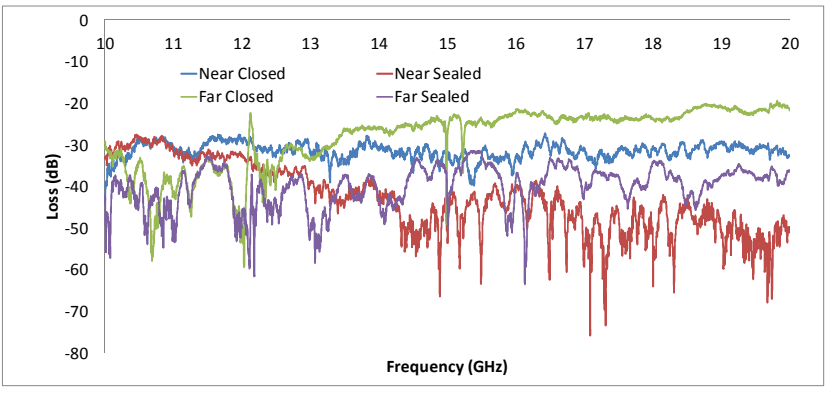

Fig. 4 - Measurement of penetration loss through the Far and Near gaps in the range $10-20 \mathrm{GHz}$

For comparison with the near case in the delay domain, the Far case found the loss of the shortest path to be $22 \mathrm{~dB}$ with the door open due to the reflection off the door frame. Note that this is additional loss to the free space loss causing significant attenuation as a result of the substantially rough surface that exists at mmWave frequencies in this instance based on the Rayleigh criterion [6]. When the door was closed, the additional loss was around $3 \mathrm{~dB}$ through the gap, while when sealing the door a further $20 \mathrm{~dB}$ of loss was measured in this instance resulting in a total penetration loss of $45 \mathrm{~dB}$. Note that though the total loss is the same as the Near case, the loss without the seal is only $25 \mathrm{~dB}$ as opposed to $33 \mathrm{~dB}$, hence an $8 \mathrm{~dB}$ improvement. Given that the difference in free space path loss between Near and Far would be negligible in a full end to end link, it is therefore more favourable to rely on the reflection from the far end of the window frame than to rely on the diffraction at the near end.

Like the Near case, the measurements for the Far case converge below $11 \mathrm{GHz}$ as shown in Fig. 4. To determine why and the phenomenon taking place, it is useful to undertake simulations to analyse the electromagnetic wave behavior through the gap at a high and low frequency in this range. This was achieved using electromagnetic dipole sources, conducting blocks and the gap width of $8 \mathrm{~mm}$. The simulation software used was Computer Simulation Technology Microwave Studio. This enabled simulations to be carried out with the same gap at different frequencies whereby the electromagnetic wave propagation in the time domain could be analysed as shown in Fig. 7. Two frequencies of interest are shown in Fig. 7 (a) at $11 \mathrm{GHz}$, and at $18 \mathrm{GHz}$ in Fig. 7 (b). Note also that the simulation includes the outer conducting ridge on the door frame as seen in Fig. 2 (b), which provides a conducting plane for specular reflections before waves enter the gap and thus the propagation scenario here is a scattering of rays through the gap with a zig zag path, which is not causing a waveguiding effect like the Near case. A further ridge inside allows the rays to reflect towards the receiver.

At $10 \mathrm{GHz}$, in free space, the half wavelength is approximately $15 \mathrm{~mm}$, while at $18 \mathrm{GHz}$ it is $8 \mathrm{~mm}$. Therefore when the half wavelength is less than $10 \mathrm{~mm}$ above $15 \mathrm{GHz}$, it is possible that zig zag standing waves could form within the gap allowing propagation, while down near $10 \mathrm{GHz}$ they cannot form in this way as the gap is too narrow for such zig zag waves. Therefore propagation is consistently weak whether sealed or not. Comparing Fig. 7 (a) and (b) shows that at $11 \mathrm{GHz}$, the case that there are no clear stationary points from which the waves can reflect reliably as a zig zag while for $18 \mathrm{GHz}$, this is much clearer where clear maximum and minimum points follow the zig zag pattern and propagate well to the other side. Note as well that above $15 \mathrm{GHz}$, the far case has even more pronounced improvement in penetration when not sealed, in the order of $10 \mathrm{~dB}$ better, while when sealed, there is still improvement but it is more frequency selective.

For higher frequencies above $20 \mathrm{GHz}$, it is inevitable that the $10 \mathrm{~mm}$ and $8 \mathrm{~mm}$ gaps are sufficiently wide enough to allow propagation. It has also been established that the Far case is more favourable and so only results of the Far case will be analysed at higher frequencies. Fig. 5 analyses the measurements of the Far case in the $22-30 \mathrm{GHz}$ range, while Fig. 6 covers the $50-67 \mathrm{GHz}$ range. Analysis of measurements in Fig. 5 shows that the improvement penetration is still achieved without the seal. However, the limitations on dynamic range due to only one amplifier being available show that there is more significant noise on the measurements after the door is closed, that it is difficult to 
obtain a reliable measure of the effect of the gap in the door frame with and without the seal. It can however be observed that measurements with the door open are shown and the frequency selectivity is substantial due to the roughness of the surface on the frame becoming more profound.

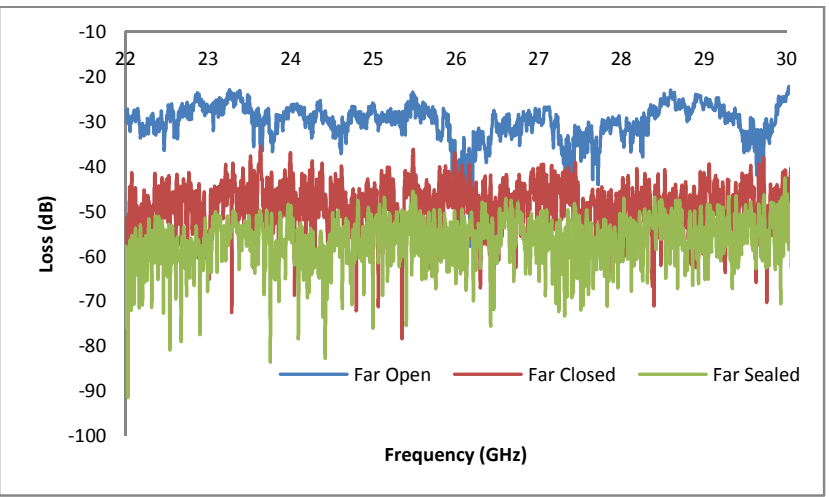

Fig. 5 - Measurement of penetration loss through the Far gaps in the range $22-30 \mathrm{GHz}$

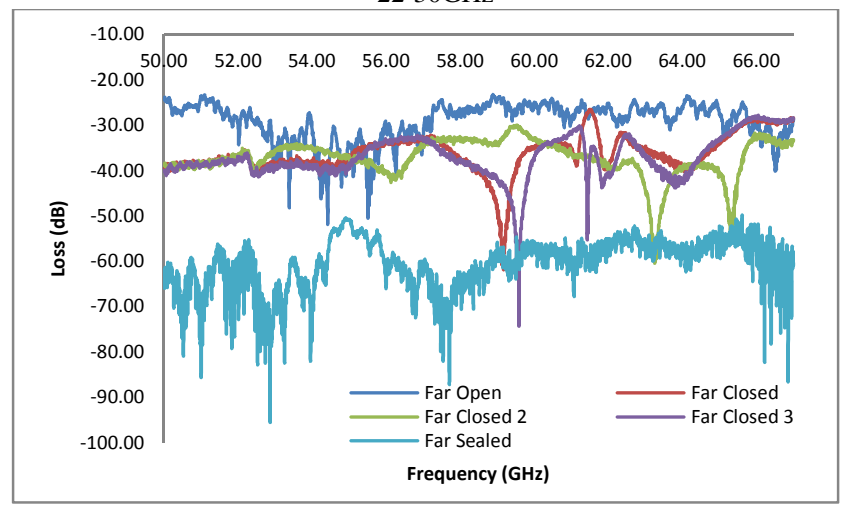

Fig. 6 - Measurement of penetration loss through the Far gaps in the range $50-67 \mathrm{GHz}$.

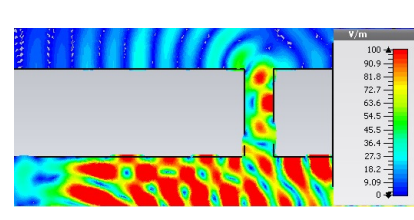

(a)

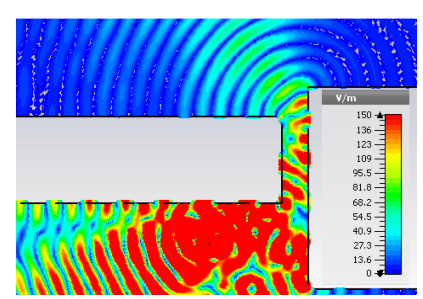

(c)

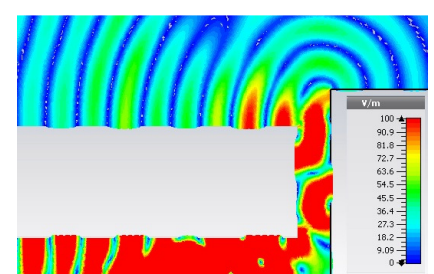

(b)

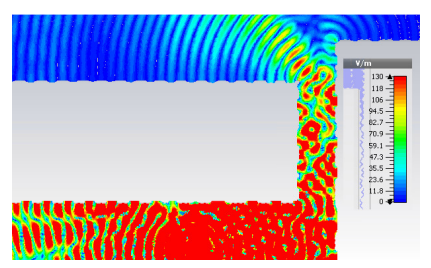

(d)
Fig. 7 - Simulation results of the Far gap at (a) $11 \mathrm{GHz}$, (b) $18 \mathrm{GHz}$, (c) $26 \mathrm{GHz}$ and (d) $60 \mathrm{GHz}$.

Measurements in Fig. 6 for the $50-67 \mathrm{GHz}$ range show that there is consistent effect of multipath when the door is open with high frequency selectivity but for the case of when the door is closed, three separate repeated measurements were taken in this case whereby the door was re-opened and then closed before each of the three measurements. Clearly there is inconsistency in results for the three measured cases, particularly beyond $56 \mathrm{GHz}$. With decreasing wavelengths, the position of the door in relation to the door frame's detailed structure will be different in the order of one millimetre. This results in the multipath conditions for such frequencies, where the half wavelength is less than $2.6 \mathrm{~mm}$, will change with small door movements and be highly frequency selective. Therefore it is clearly desirable at higher frequencies to have consistent structures of gaps in buildings to enable more predictable penetration. Nonetheless at such high frequencies it is clear that as the gap has become more electrically large, as much as $20 \mathrm{~dB}$ improvement in penetration can be achieved due to the gap when comparing with the sealed case.

Finally for completeness of results, the simulated results in the Far case for $26 \mathrm{GHz}$ and $60 \mathrm{GHz}$ are plotted in Fig. 7 (c) and (d) respectively. It is clear that standing waves at $26 \mathrm{GHz}$ are even more profound than what was seen at $18 \mathrm{GHz}$ where nulls and maximas can be identified in a single zig zag. Furthermore, at $60 \mathrm{GHz}$, the gap has become so electrically wide that it has formed a multipath fading scenario, which reflects the frequency selective fading seen in measurements.

\section{CONCLUSION}

Measurement results at mmWave for outdoor to indoor have been presented that compare the effect of relying either on diffraction off a near edge of a window or diffuse scattering from a reflection off the far edge of a window or door frame. Measured results show that it is more beneficial to exploit the reflection off the far end of the frame, but use of gaps in infrastructure can bring substantial benefit. Results presented in this paper indicate that exploitation of gaps in building infrastructure can improve the penetration loss in the range of $10-20 \mathrm{~dB}$ where it performs best at higher frequencies or with wider gaps that are electrically large enough for the frequency of operation. Smooth gap structures are however required to avoid multipath effects within the gap that is being propagated through.

\section{REFERENCES}

[1] W. Roh, J-Y Seol, J. Park, B. Lee, J. Lee, Y. Kim, J. Cho, K. Cheun, F. Aryanfar, "Millimeter-wave beamforming as an enabling technology for $5 \mathrm{G}$ cellular communications: theoretical feasibility and prototype results", IEEE Communications Magazine, vol. 52, issue 2, February 2014, pp106-113.

[2] "Technical feasibility of IMT in bands above $6 \mathrm{GHz}$ ", International Telecommunication Union, Report M.2376, July 2015.

[3] J. B. Andersen, T. S. Rappaport, Y. Yoshida, "Propagation measurements and models for wireless communications channels", IEEE Communications Magazine, vol. 33, issue 1, January 1985, pp42-29.

[4] T. S. Rappaport, S. Sun, R. Mayzus, H. Zhao, Y. Aszar, K. Wang, G. N. Wong, J. K. Schulz, M. Samimi, F. Gutierrez, "Millimeter Wave Mobile Communications for 5G Cellular: It Will Work!", IEEE Access, vol. 1, 2013, pp335-349.

[5] H. Zhao, R. Mayzus, S. Sun, M. Samimi, J. K. Schulz, Y. Azar, K. Wang, G. N. Wong, F. Gutierrez, T. S. Rappaport, "28 GHz millimeter wave cellular communication measurements for reflection and penetration loss in and around buildings in New York city", IEEE International Conference on Communications, 2013, pp5163-5167.

[6] S. R. Saunders, A. Aragon-Zavalá, "Antennas and Propagation for Wireless Communication Systems", $2^{\text {nd }}$ Edition, 2007, Wiley, UK.

[7] A. W. Scott, "Understanding Microwaves", 2005, Wiley, USA. 